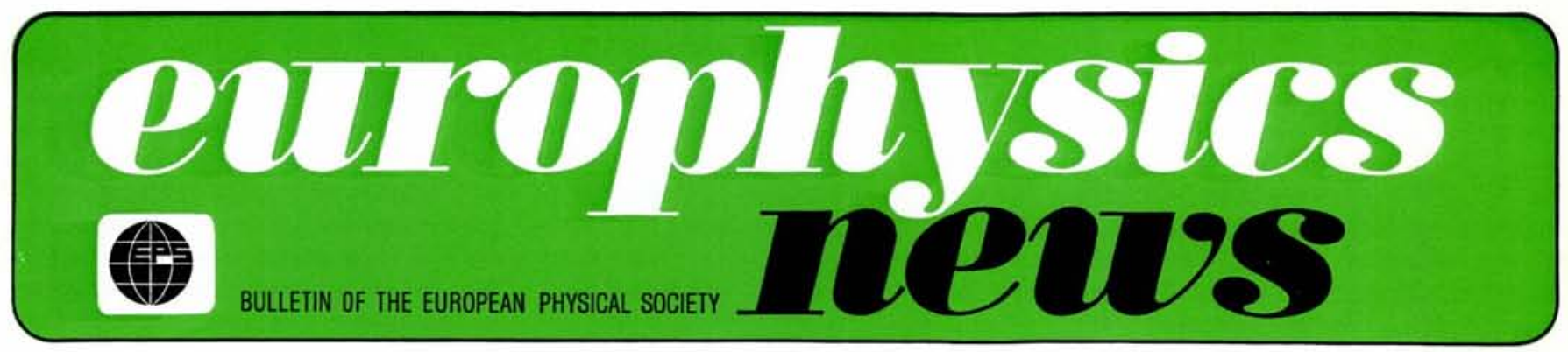

J.A.

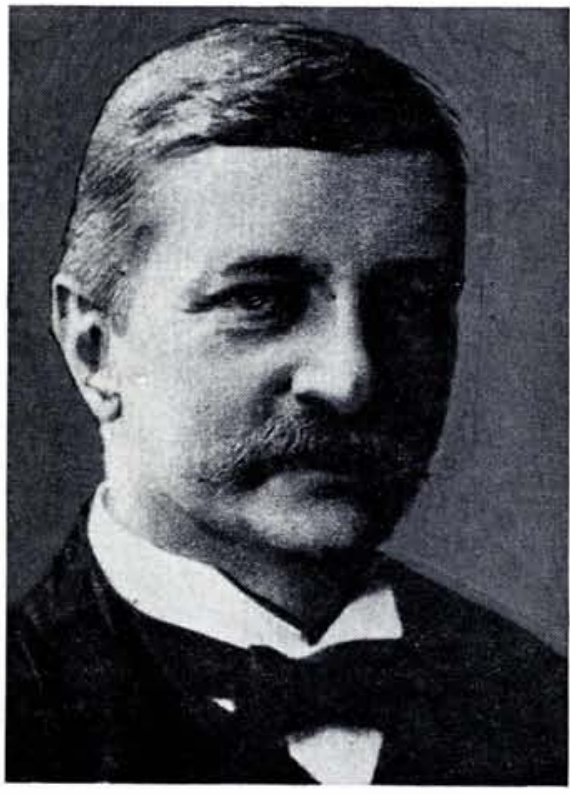

Professor Janne R. Rydberg of Lund (18541919).

The study of atomic Rydberg states is an old story as the first qualitative observations on series in alkali spectra appeared in a paper published by Liveing and Dewar in 1879. Eleven years later, Rydberg proposed his famous formula for the wave numbers of a spectral series : $\sigma=C-R /(n+\delta)^{2}, n$ being a running integer, $\delta$ a number approximately constant for the high members (large values of $n$ ) of a given series, and $R$, a universal constant common to all series in all spectra of neutral atoms. The decisive advances in the understanding of atomic Ryd-

\section{Contents}

What's New about Atomic Rydberg States

Earthquake Source Physics

Optics and Solid State at Laboratories RCA, Zurich

EPS Scholarships

$11 \& 12$

Society News

\title{
What's New about Atomic Rydberg States?
}

\section{S. Feneuille, Orsay}

(Aimé Cotton Laboratory)

berg states was, of course, provided by Quantum Mechanics and from the very first calculations of Schroedinger in 1926 on atomic hydrogen, it was possible to predict the main properties of highly excited atomic Rydberg states: atoms in states corresponding to large values of $n$ have a very large size (the atomic radius varies as $n^{2}$ ); their radiative lifetime is very long (this quantity varies as $n^{3}$ ) ; they are highly polarizable by an electric field (the scalar polarizability varies as $n^{7}$ ) ; they can exhibit a strong diamagnetism (the diamagnetic contribution to the Zeeman effect varies as $n^{4}$ ) ; they are easily destroyed by collisions (the geometrical area of the atom varies as $n^{4}$ ). Then, the origin of the quantum defect, $\delta$ was rapidly understood, as also the deviations observed with respect to the Rydberg formula in many electron spectra (the so-called perturbed series). After this "golden time", while atomic spectroscopy and more generally atomic physics of valence states (small values of $n$ ) became a more and more active and fruitful field, for Rydberg states, the situation remained approximately the same for forty years, with the exception of a few exciting observations on the foreign gas broadening and shift of high members of alkali spectral series (the so-called Amaldi-Segré anomalies explained by Fermi) and some very nice experiments on strong magnetic field effects. This lack of rapid progress came essentially from the experimental impossibility of producing Rydberg atoms in an efficient and selective way, and up to a few years ago, experimental data on Rydberg states were almost all obtained from absorption spectra exactly in the same way as in the days of Rydberg. Not entirely however, as in 1965 an emission line at $5.4 \mathrm{GHz}$ was detected coming from the Orion nebula and attributed to a transition between the levels $n=110$ and $n=109$ of atomic hydrogen. Numerous other observations showed that Rydberg atoms are rather common species in interstellar space but it is still not very easy to respond to the behaviour of objects so far away.

\section{Revolution Provoked by Tunable Lasers}

As in many sub-fields of atomic physics, the situation became radically different with the advent of tunable lasers, and since 1975, the number of research groups working on atomic Rydberg states and therefore the number of papers published on this subject has been continuously increasing. Today, tunable lasers play a double role in the revolution. First, thanks to the light power they can deliver, they allow one, through multiphoton processes or multistep excitation, to populate efficiently highly excited Rydberg states $(n \leq 100)$ either from the ground state or from metastable states and thus, to study transitions not allowed in conventional one-photon absorption techniques. Second, it is now possible to produce Rydberg atoms not only in vapours but also in atomic beams. This new possibility has two main consequences. On the one hand, in an atomic beam, Rydberg atoms are not destroyed by collisions and, because of their long radiative lifetime (of the order of $1 \mathrm{~ms}$ for $n=$

Europhysics News is published monthly by the European Physical Society. (c) 1979. Reproduction rights reserved. 
$50)$, a rather long time is available to study their properties in the presence of various perturbations. On the other hand, the homogeneous width of a Rydberg level (which mainly results from collisions in vapours) is reduced to the radiative natural line width which is extremely small (a few $\mathrm{kHz}$ for $n=50$ ). One can therefore, take advantage of ultra high resolution techniques of laser or microwave spectroscopy.

In fact, the first contributions brought by laser techniques concerned the spectroscopy of Rydberg states. Very numerous data have been obtained essentially in alkalis (using Doppler-free ultra-high resolution methods) and in alkaline earths (using mainly Doppler limited spectroscopic techniques). In all cases, atomic resonances can no longer be detected by measuring light absorption, and detection by fluorescence becomes less and less efficient when $n$ increases. New non-optical detection methods had thus to be carried out. All of them utilize the fragility of Rydberg states under the influence of collisions or of a static electric field. In particular, Rydberg atoms in vapours are easily ionized by atomic collisions and atomic resonances can be detected through the number of ions produced. This can be done in a particularly efficient way by putting the atomic sample inside a diode and measuring the current resulting from the change in the space charge. In atomic beams, the best way to detect Rydberg atoms is to put them in a static electric field. If the field strength is larger than a critical value given by:

$$
F_{c}=[2(n+\delta)]^{-4}
$$

in a.u., a Rydberg atom ionizes spontaneously. Here again, the resonance signal is obtained by counting the number of ions produced as a function of the excitation energy. More precisely, the ionization field is applied after the laser excitation pulse is finished to avoid Stark perturbation of atomic structure.

Up to now, the extensive data obtained, at relatively low resolution, in alkaline-earths or in lanthanides have not exhibited new phenomena properly speaking, but they allow in many cases a precise classification of the lines within the frame of the Multichannel Quantum Defect Theory, thus showing the validity of this parametric approach of highly excited many electron spectra. In alkalis, most of the results concern very small effects such as fine structure for example which vary as $n^{-3}$. Several anomalies have been observed on splitting and on intensity ratios, showing that, rather paradoxically, Rydberg states may have in many respects a strong non hydrogenic character and exhibit dramatic relativistic effects. Particular attention must be paid to double resonance experiments involving laser excitation followed by microwave transitions. Such experiments provide not only very precise spectroscopic data on transitions between Rydberg states (which could have, in the future, important consequences for metrology) but also, they demonstrate the possibility of using Rydberg atoms as efficient detectors of microwave radiation. In particular, superradiance on microwave transitions between atomic Rydberg states has recently been observed and maser oscillation on such transitions has been carried out in a sodium atomic beam.

Beyond the spectroscopic experiments described above, a lot of attention has recently been paid to the properties of highly excited atoms in the presence of "strong" magnetic and electric fields. Concerning magnetic fields, most of the experiments have been performed by using standard absorption techniques, just as in the pioneering work of Jenkins and Segré in 1939 on the diamagnetic shifts of sodium and potassium Rydberg states. Two-photon and two-step laser spectroscopy has also been utilized. In particular, a detailed study of the diamagnetic structure of the sodium levels around $n=28$ in a field of $6 \mathrm{~T}$ was recently carried out by laser spectroscopy on an atomic beam and successfully compared with a calculation based on a numerical diagonalization including spherical basic states from $n=25$ to $n=31$. This perturbation approach cannot be used to understand the quasi-Landau structure appearing in the vicinity - below and above - of the zero field ionization limit. This quasi-Landau structure, which has been observed in the spectra of $\mathrm{Ba}, \mathrm{Sr}$ and $\mathrm{Li}$ for field intensities varying between 2.5 and $5 \mathrm{~T}$, consists in oscillations of the photoabsorption cross-section, whose frequency is proportional to the cyclotron frequency (by a factor $3 / 2$ or $1 / 2$ ). In spite of recent theoretical advances, this structure is not yet fully understood even in the one electron approximation. It is amazing that such a simple problem has not yet found a definitive solution, but one must remember that even for hydrogen, the Zeeman Hamiltonian is not fully separable.

Apparently, the problem of an atom interacting with a static electric field might be easier to understand as the Stark Hamiltonian for the hydrogen atom is fully separable in parabolic coordinates. A few years ago, it seemed difficult to imagine new phenomena in the study of atoms in the presence of a static electric field, but, as mentioned above, field ionization was rapidly recognized as a good technique to detect Rydberg atoms. Gradually, it became more and more evident that this phenomenon was not so simple after all and several research groups in the USA and in Europe began to study it on its own account. The first experiments dealt with the Stark structure of Rydberg states in the presence of very weak fields (a few $V / c m)$. This Stark structure can be understood within the frame of quasi degenerate first order perturbation theory. This perturbation approach can no longer be used as soon as the electric field becomes intense enough for ionizing the atom. As already noticed, this critical field value varies as the square of the binding energy of the excited electron and therefore decreases rapidly when $n$ increases. This is the reason why field ionization is so easy to be observed for Rydberg states. In other words, in the presence of a static electric field, $F$, the ionization limit of an atom is lowered by a quantity proportional to $F^{1 / 2}$. More precisely, this new limit is given in atomic units by :

$$
E_{c}=E_{0}-2 F^{1 / 2}
$$

$E_{\text {o }}$ being the ionization energy of the free atom.

The interpretation of this phenomenon is actually very simple as $E_{c}$ is nothing else but the energy of the saddle-point which appears in the potential energy surface of an electron interacting simultaneously with a central Coulomb field and a uniform field. More unexpected was the appearance of very narrow resonances in the photoionization spectrum of an alkali atom in the presence of a static electric field. These resonances have been observed first on a rubidium atomic beam transversely illuminated by $a$ pulsed single-mode laser. In this case, they are sufficiently narrow (width smaller than $50 \mathrm{MHz}$ ) to be isotopically selective, at least when $\sigma$ light polarization is used. A more extensive study at lower resolution has been performed on lithium and it has been confirmed that these photoionization resonances appear only for excitation energies larger than $E_{c}$ For excitation energies lower than $E_{c}$ the atomic structure reduces to quasi-stable Stark levels.

It must be emphasized that most of these photoionization resonances should not appear in hydrogen since, 
for this very peculiar atom, they would correspond to quasi-stable levels. These quasi-stable states are related to those classical orbits which miss the saddle-point despite their higher energy. Even in hydrogen, they are embedded in underlying continua, but, because of the supersymmetry connected with separability, they do not interact with those continua. As soon as the atomic potential is no longer purely coulombic, supersymmetry disappears and interaction with the continua is allowed, leading to a phenomenon quite analogous to auto-ionization in many electron spectra or to predissociation in molecules. This ionization mechanism occurring through the excitation of a quasi-stable state was understood only one year ago. Experimental proof of its validity was recently given by the observation of characteristically asymmetric resonance peaks (so-called Fano profiles) in the $\Pi$-photoionization spectrum of atomic rubidium in the presence of a static electric field.

Other interesting features also appear in this type of photoionization spectrum. In particular, the photoionization cross-section exhibits fielddependent oscillations even for excitation energies higher than the ionization energy of the free atom. These oscillations, which have been recently understood in the one-electron approximation, are tightly connected with the oscillations appearing in photoabsorption or photoreflectance spectra of semiconductors or insulators in the presence of a static electric field. All the observed phenomena seem to be understood at least qualitatively, but up to now, all the experiments have been realized on alkalis and it is not obvious that the situation will remain exactly the same for many electron spectra, especially in Rydberg series perturbed by doubly excited valence states. Moreover, exciting predictions have been made for Rydberg atoms interacting with crossed electric and magnetic fields. Further experiments are certainly needed and one can easily guess that this subject will remain very active in the future.

\section{Collisional Processes}

Collisional properties of Rydberg atoms have also given rise to various experimental and theoretical studies. Many of them concern collisional depopulation of Rydberg states. The experimental technique consists essentially in recording, as a function of time, the fluorescence emitted from a Rydberg state selectively excited by a short laser pulse. Therefore, it can be applied to moderately excited $(n<30)$

\section{NATO ADVANCED STUDY INSTITUTE}

5-16 May, 1980

Lillehammer Turisthotell, Norway

\section{Exploration of the Polar Upper Atmosphere}

Subject Areas :

Neutral polar atmosphere above the tropo- Solar-magnetosphere-polar atmosphere interpause

Polar ionosphere ( $F$ region)

Polar ionosphere (D \& E regions)

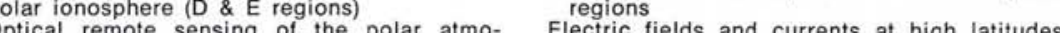
sphere and ionospher actions/auroral morphology regions \& micropulsation in the polar Electric fields and currents at high latitudes Applications of polar exploration

Historical exploration: long-term aspects of polar geophysics

For information, contact Prof. A. Egeland, Institute of Physics,

University of Oslo, РОВ 1038

N - Blindern, Oslo 3

Deadline for applications: 1 February, 1980 species only. Many systems including mainly alkalis and noble gases have been investigated. Two main features are to be emphasized. On the one hand, one observes, for rather low values of $n$ a rapid increase with $n$ of quenching cross-sections, especially when Rydberg states are quenched by their ground state counterpart. On the other hand, it appears that, under the influence of collisions with unexcited noble gas atoms, the apparent lifetime of some alkalis' Rydberg states, with low orbital angular momentum values, are enormously lengthened. This rather paradoxical effect has been explained by a quasi-elastic process induced by a short range interaction between the quasi-free Rydberg electron and the collision partner. This process produces a strong mixing with states having higher orbital angular momenta (and thus, longer lifetimes) but very close in energy.

Many other types of collisional processes have also been studied. Perhaps the most exciting concerns the production of negative molecular ions in collisions between polar molecules and Rydberg atoms. In such a process, one takes full advantage of the very small kinetic energy of the Rydberg electron. In fact, the phenomenon can be quantitatively understood within the frame of the independent particle model, the atomic core acting only as a passive spectator. Therefore, the use of Rydberg atoms appears as a very simple and efficient trick to produce quasi-free electrons with very low energies. Moreover, it has been shown that the small energy needed to ionize the Rydberg atom comes mainly from a transfer between atomic electronic energy and molecular rotation energy. Again, such a process is specific to Rydberg states, and it opens fascinating perspectives in chemical physics.

In conclusion, during the past few years, considerable and often unexpected advances have been made in our understanding of the properties of Rydberg atoms. As liquid crystals in the physics of condensed matter, this is a beautiful example of a very old subject which has been considered for many years as fully completed and which, nevertheless, has been renewed in a very short time. Various applications concerning for example laser isotope separation, microwave detectors or infrared lasers have already been suggested. From a more fundamental point of view, many new phenomena have been observed and understood. However, some problems are still open, and one can easily predict that for some time to come, the study of Rydberg states will certainly remain a very active sub-field of atomic physics.
8-12 September, 1980

Lausanne (Switzerland)

\section{2nd International Meeting on the Small Particles and Inorganic Clusters}

Topics: Structure, nucleation, dynamical properties of the lattice, electronic properties, beams of clusters, theoretical description, catalysis, applications.

Advisory Committee : Profs. J. Friedel (Paris), O.F. Hagena (Karlsruhe), R. Kern (Marseille), W.D. Knight (Berkeley), R. Kubo (Tokyo), R.P. Messmer (Schenectady, N.Y.), B. Mühlschlegel (Cologne).

Abstracts to be submitted by 31 January, 1980 to :

Prof. J.-P. Borel, EPFL, 33 Ave. de Cour, CH - 1007 LAUSANNE 